\title{
A Study of Clinical Profile and Factors Associated with Cerebrospinal Fluid Rhinorrhea with Outcomes of Various Treatment Modalities at Tertiary Healthcare Center
}

\author{
Dhruvika M Rathva ${ }^{1}$, Rahul R Gupta ${ }^{2}$, Ranjan G Aiyer ${ }^{3}$, Amey P Patankar ${ }^{4}$
}

\begin{abstract}
Introduction: Cerebrospinal fluid (CSF) rhinorrhea implies an abnormal communication between the subarachnoid space and the nasal cavity leading to drainage of CSF to the exterior.

Aims and objectives: To study the clinical profile and factors associated with CSF rhinorrhea with outcomes of various treatment modalities at a tertiary healthcare center.

Materials and methods: This study was conducted in the Department of ENT and Head-Neck Surgery, SSG Hospital and Government Medical College, Vadodara, Gujarat, from April 2014 to November 2016. Patients were selected from those attending the outpatient department (ENT/ neurosurgery OPD), indoor patients (admitted at ENT ward 19/general surgery/neurosurgery wards), and emergency department of the hospital during the study period.

Results: The age of patients ranged from 18 to 70 years with a mean age of 38.6 years; 12 (60\%) were males and 8 (40\%) were females. A total of 20 patients of CSF rhinorrhea were included in our study. Majority of rhinorrhea patients presented with watery nasal discharge as their primary complaint, which increased on straining or bending forward. The most common etiology was posttraumatic in $55 \%$ followed by iatrogenic in $25 \%$ and spontaneous in $20 \%$. Treatment modalities used were: (A) conservative measures done in 50\%, (B) endoscopic endonasal CSF leak repair in $35 \%$; (C) repair by the craniotomy approach done in $15 \%$. The CSF leak was successfully repaired by the endoscopic endonasal approach in six (86\%) patients. About $71 \%$ of patients who were put on conservative measures responded favorably in the 1 st week only and the rest $29 \%$ needed 2 weeks for stoppage of CSF rhinorrhea.

Conclusion: Cerebrospinal fluid rhinorrhea has varied etiology. Mostly they are posttraumatic following road traffic accidents. Early identification of CSF rhinorrhea is important and can be safely managed conservatively in majority of cases.

Keywords: Cerebrospinal fluid leak, Cerebrospinal fluid rhinorrhea, Endoscopic endonasal cerebrospinal fluid leak repair.

Clinical Rhinology An International Journal (2018): 10.5005/jp-journals-10013-1342
\end{abstract}

\section{INTRODUCTION}

Cerebrospinal fluid (CSF) rhinorrhea implies an abnormal communication between the subarachnoid space and the nasal cavity leading to drainage of CSF to the exterior. Having knowledge about these leaks, how they occur, and how to treat them is important for multiple reasons, most important being risk of intracranial infection-if such leaks persist for long time.

Nowadays, otorhinolaryngologists have a major role in the evaluation and management of anterior and lateral skull base defects that result in CSF leaks. Currently, most of such leaks located in the anterior skull base can be jointly treated by otorhinolaryngologists and neurosurgeons using the endoscopic endonasal approach. Many a time such leaks resolve with nonsurgical management also. There are many causes of CSF rhinorrhea. They can be traumatic, spontaneous, iatrogenic, congenital, or neoplastic. Cerebrospinal fluid leaks may present a significant challenge in diagnosis, localization of defect, and ultimate management. Thus, otorhinolaryngologists should be aware of the various diagnostic and treatment options available in order to manage such patients.

In 1745, Bidloo the Elder was the first to describe CSF rhinorrhea after a traumatic skull fracture and correlate them. ${ }^{1}$ The CSF leak was first described by Galen in the second century as a normal, periodic release of CSF into the nose via the sella and ethmoid regions. This process was accepted as a physiological communication
'Department of ENT and Head-Neck Surgery, Parul Institute of Medical Sciences and Research, Vadodara, Gujarat, India

2,3 Department of ENT and Head-Neck Surgery, Baroda Medical College and SSG Hospital, Vadodara, Gujarat, India

${ }^{4}$ Consultant neurosurgeon, Department of neurosurgery, Baroda medical collage and SSG Hospital, Vadodara, Gujrat, India

Corresponding Author: Dhruvika M Rathva, Department of ENT and Head-Neck Surgery, Parul Institute of Medical Sciences and Research, Vadodara, Gujarat, India, Phone: +91 9664577016, e-mail: dhruvikamr@gmail.com

How to cite this article: Rathva DM, Gupta RR, Aiyer RG, et al. A Study of Clinical Profile and Factors Associated with Cerebrospinal Fluid Rhinorrhea with Outcomes of Various Treatment Modalities at Tertiary Healthcare Center. Clin Rhinol An Int J 2018;11 (2 and 3):36-39. Source of support: Nil

Conflict of interest: None

for many centuries until the late 1800s, when St. Clair Thompson described a series of patients with an abnormal, nontraumatic condition that he termed rhinorrhea. ${ }^{2}$ Although traumatic leakage of CSF is overwhelmingly more common, the first published case of CSF rhinorrhea was a nontraumatic high-pressure type due to hydrocephalus reported by Miller in $1826,{ }^{3}$ followed by reports by King in 1834. Neurosurgical treatment of such dural fistulas began much later with the work of Grant and Dandy. Dandy first reported 
the successful closure of rhinoliquorrhea in $1926 .{ }^{4}$ He performed a frontal craniotomy and used fascia lata to occlude the defect. Three cases of traumatic leakage treated surgically were reported in 1927 by Cushing. The first series of cases treated by transcranial extradural repair using fascia lata was published by Cairns in $1937 .{ }^{5}$ The intradural repair technique was first used by Taylor and reported by Eden in 1941. In 1944, Schroeder described a patient with CSF rhinorrhea who recovered after treatment with sulfonamides and a lumbar puncture. ${ }^{6}$

\section{Materials and Methods}

This study was conducted in the Department of ENT and Head-Neck Surgery, SSG Hospital and Government Medical College, Vadodara, Gujarat, from April 2014 to November 2016 after being approved by the Institutional Scientific and Ethical Committee. Patients were selected from those attending outpatient department (ENT/ neurosurgery OPD), indoor patients (admitted at ENT ward 19/ general surgery/neurosurgery wards) and emergency department of the hospital during the study period. They were enrolled in the study after taking an informed written consent in the vernacular language. A total of 20 patients of CSF rhinorrhea were included in our study. On admission, the demographic profile of patients as well as a detailed clinical history were noted. Thorough history was elicited of each and every patient, to find out any associated etiological factors (i.e., H/o trauma, previous surgery, any tumor). In iatrogenic cases, details of surgery and any complication during surgery were obtained. Detailed history including treatment history, past history, and family and personal histories were obtained. In all our patients, clinical examination was done with complete general examination and gross systemic examination. We also checked for any comorbidities. This was followed by detailed ear, nose, and throat examination. In all CSF rhinorrhea suspected patients, nasal endoscopy was performed under strict asepsis. It was done to look for the site of leakage and any nasal pathologies or abnormalities. The handkerchief test was performed in all cases suspected for CSF leaks. It dried out without stiffening in CSF leak cases. The halo sign was seen in few posttraumatic cases. All cases were investigated with general hematological and biochemical tests. Suspected leaking of CSF nasal fluids were checked for cell counts, protein, and sugar examination. $\beta-2$ transferrin in the nasal discharge was not assessed in this study, although being most specific for CSF. The reasons being its inability to identify the site of leak and also nonavailability of this test at our hospital.

In majority of our cases, CT cisternography was performed to confirm our diagnosis, except few cases in whom there was intraoperative leak. CT cisternography was performed using intrathecal lohexol injection. All patients were then placed in a prone kneeling or prone Trendelenburg position for 2-5 minutes to facilitate opacification of the cisterns. No other provocative maneuvers were used. The patients immediately underwent CT in the axial and coronal planes using parameters that were identical to those of the noncontrast examination. lohexol is a secondgeneration nonionic radiocontrast agent. In all posttraumatic cases posttrauma CT head evaluated for any major defects and/or fracture lines. Intraoperative intrathecal application of fluorescein was not done in any of our patients.

Our treatment started with conservative management. All patients were advised bed rest and head end elevation up to $45^{\circ}$. Stool softeners in form of oral laxatives were given to high-risk patients to avoid straining. All patients were kept under higher injectable antibiotics coverage. A few patients responded to these measures and leak stopped. In a minor percentage of our patients who didn't respond to such general measures and persisted with CSF leak, a lumbar drain was inserted. All the patients who did not respond to all these conservative measures were taken up for surgery. The patients who were considered as high-risk cases (one posttraumatic case with large defect and one postfrontal craniotomy patient who had developed CSF rhinorrhea) by the neurosurgeon were taken up for the external approach. All the rest were taken up for endonasal endoscopic CSF leak repair. All patients were followed up regularly.

\section{Results}

The age of patients ranged from 18 to 70 years with a mean age of 38.6 years; among them, 12 (60\%) were males and 8 (40\%) were females. Apart from 3 (15\%) patients (who developed iatrogenic intraoperative CSF leak), all others $(17,85 \%)$ presented with watery nasal discharge, which increased on straining or bending forward. One of our patients presented with watery nasal discharge with fever and vomiting.

Among all these CSF rhinorrhea patients, 11 patients had posttraumatic ( 9 patients of road traffic accident, 1 patient after fall from height, 1 with assault) CSF leaks, 4 patients developed spontaneous CSF leaks, and 5 patients had iatrogenic CSF leaks (Table 1). Two patients had leak following frontal craniotomy, and rest three had CSF leak during resection of nasal mass involving the anterior skull base [one malignant melanoma, one esthesioneuroblastoma, and one sellar space occupying lesion (SOL)].

The most common site of CSF leak was found to be the cribriform plate of ethmoid bone (Fig. 1) followed by frontal bone (Fig. 2), sphenoid, ethmoid bone, and diaphragma sellae (Table 2).

A total of 10 (50\%) out of 20 patients responded to conservative measures and relieved. A total of 7 (35\%) out of 20 patients were taken up for endoscopic endonasal CSF leak

Table 1: Distribution of patients according to etiology

\begin{tabular}{lc}
\hline Etiology & No. of patients $(\%)(n=20)$ \\
\hline Posttraumatic & $11(55)$ \\
latrogenic & $5(25)$ \\
Spontaneous & $4(20)$ \\
\hline
\end{tabular}

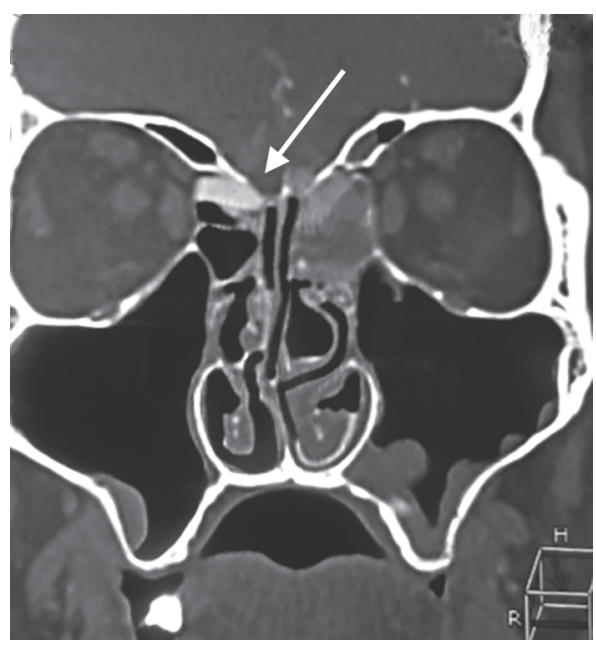

Fig. 1: Site of leak—right cribriform plate 


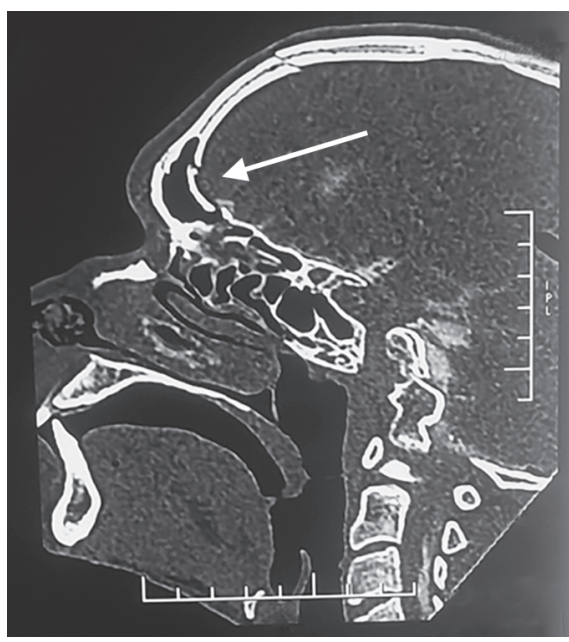

Fig. 2: Posterior wall of frontal sinus

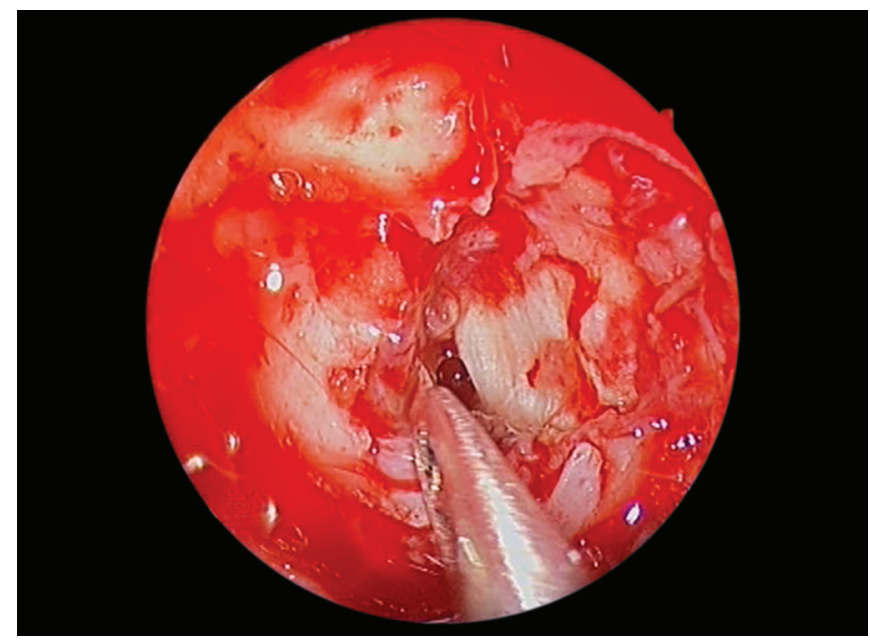

Fig. 4: Intraoperative site of leak identified by Valsalva maneuver-roof of sphenoid

Table 2: Distribution according to site of CSF leak

\begin{tabular}{lc}
\hline Site of leak & No. of patients $(\%)(n=20)$ \\
\hline Cribriform plate area & $11(55)$ \\
Frontal & $3(15)$ \\
Sphenoid & $3(15)$ \\
Ethmoid & $2(10)$ \\
Diaphragma sellae & $1(05)$ \\
\hline
\end{tabular}

repair and 3 (15\%) out of 20 patients were operated by the open craniotomy approach.

Among 11 posttraumatic CSF leak patients, 7 (64\%) patients were resolved with conservative measures, $2(18 \%)$ patients were operated by endoscopic endonasal CSF leak repair, whereas 2 (18\%) patients were operated by the frontal craniotomy approach (Fig. 3).

Among three spontaneous CSF leak patients, two (67\%) patients resolved with conservative measures and one (33\%) patient was operated by endoscopic endonasal CSF leak repair.

Two patients, out of five iatrogenic cases, had CSF leak after frontal craniotomy for pituitary lesions. Those patients had CSF leak after 2-3 days of surgery. In three out of five iatrogenic cases,

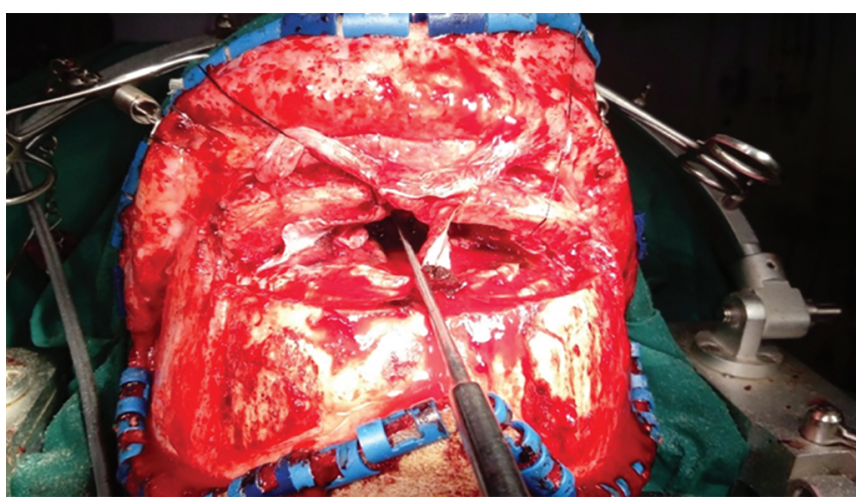

Fig. 3: Identification of leak site-by frontal craniotomy approach

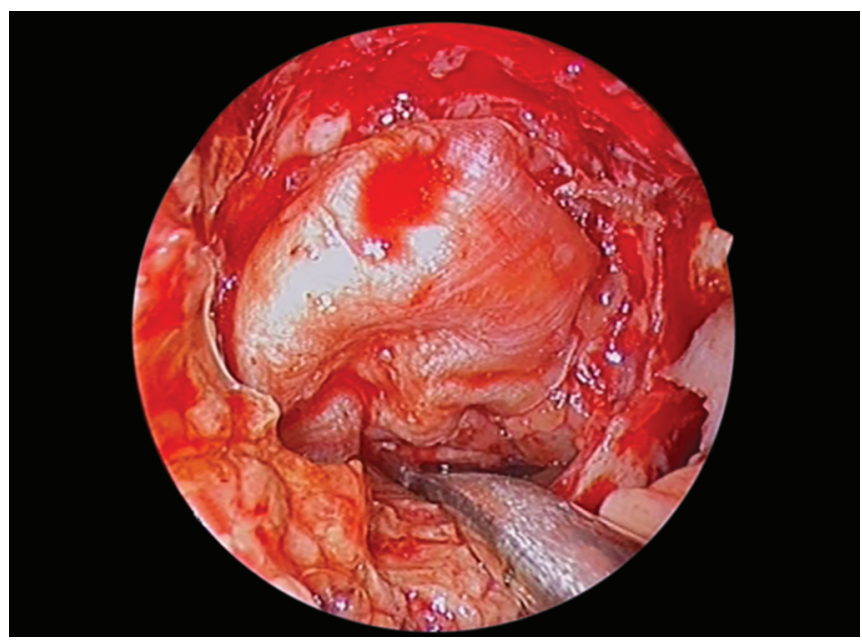

Fig. 5: Leak site covered with fascia lata

leak was identified intraoperatively (Fig. 4) during resection of nasal mass involving the anterior skull base (one malignant melanoma, one esthesioneuroblastoma, and one sellar SOL). Such leaks were repaired at same time using different grafting materials like fat, fascia lata, and cartilage. Figure 5 showing defect covered with fascia lata.

Among, five iatrogenic CSF leak patients, four (80\%) patients were operated by endoscopic endonasal CSF leak repair. One (20\%) patient, who was previously operated by frontal craniotomy for large pituitary lesion, was again operated by the frontal craniotomy approach to repair CSF leak.

The CSF leak was successfully repaired by the endoscopic endonasal approach in six (86\%) patients. One patient (16\%) had recurrent leak again after 1 month. This patient was obese and hypertensive with high pressure leak. This patient was treated with lumboperitoneal shunt insertion.

Time to respond to conservative measures in posttraumatic cases: $2-7$ days required in $71 \%$ cases and $8-14$ days in $29 \%$ cases.

\section{Discussion}

All our patients $(85 \%)$ came with complaint of clear watery nasal discharge apart from three in whom iatrogenic leak was identified intraoperatively. This is consistent with the observation by Schlosser et al. ${ }^{7}$ that the most frequent clinical manifestation of CSF rhinorrhea is watery nasal discharge, with most patients not complaining of headache. 
In all CSF rhinorrhea cases, other than iatrogenic cases, radiological diagnosis by $\mathrm{CT}$ cisternography confirmed the exact site of leak. Vishwakarma et al. ${ }^{8}$ showed that the radiological diagnosis by CT cisternography confirmed the CSF leaks and located the defect in 15/15 (100\%) patients.

In our series, the most common site of CSF leak was cribriform plate area (55\%) followed by the sphenoid sinus (15\%), frontal sinus (15\%), ethmoid sinus (10\%), and diaphragma (5\%). This is similar to the findings of Vishwakarma et al., ${ }^{8}$ and Lindstrom et al., ${ }^{9}$ who noted the cribriform plate to be the most common site, followed by ethmoid and sphenoid sinus. Mishra et al. ${ }^{10}$ and Mohammadi et al. ${ }^{11}$ noted that the most common site of leak was the left cribriform plate area (61\%). In our study of 10 posttraumatic CSF leak patients, 7 (70\%) patients responded to conservative measures. Rest 30\% patients were managed by surgical modalities.

In similar study, Bell and colleagues reported that nonsurgical measures alone provided resolution in $85 \%$ of the 34 cases. Only three (33\%) of the nine patients with posttraumatic CSF rhinorrhea did not respond to conservative measures and required surgical intervention. In our study, the rate of successful endoscopic CSF leak repair was $86 \%$. In a similar study, Presutti reported a success rate of $88.5 \%$ on the first attempt.

The success rate after endoscopic transnasal repair of CSF rhinorrhea in similar studies noted was: Burns et al., 1996: 83\%.

As the literature reveals, spontaneous leaks have the highest recurrence rate after surgical repair to the tune of $25-87 \%$. In comparison, less than $10 \%$ recurrence rate is seen in CSF leaks of other etiologies. ${ }^{12,13}$ In one of our patients, there was CSF leak from the cribriform area, but the exact site could not be identified precisely during surgery and hence could have been the cause of failure. This patient was later treated by the L-P shunt with successful results.

In a similar study, Clark et al. ${ }^{14}$ used the L-P shunt in a spontaneous CSF rhinorrhea case with benign intracranial hypertension. It responded well to the same and resolved. In our study, one patient had intraoperative CSF leak from diaphragma sellae who underwent transnasal endoscopic pituitary surgery. The usual cause of CSF rhinorrhea during pituitary surgery is trauma to the diaphragma resulting from instruments like curette, forceps, or suction. The diaphragma may often be very thin and susceptible to trauma. Also in front of the infundibulum, the upper aspect of the gland is related directly to arachnoid and pia. The subarachnoid space here extends below the diaphragm and may be inadvertently breached while removing the tumor. Cappabianca and colleagues ${ }^{15}$ report an intraoperative CSF leak in 90 of their 242 patients (37.1\%) who underwent transnasal endoscopic pituitary surgery. Lorenz et al. $^{16}$ reported a CSF leak rate of $8.3 \%$ after resection of 24 hypophyseal tumors.

\section{Conclusion}

Cerebrospinal fluid rhinorrhea has varied etiology. Mostly they are posttraumatic following road traffic accidents. Early identification of CSF rhinorrhea is important and can be safely managed conservatively in majority of cases. The endoscopic endonasal approach gives successful results with precarious craniotomies being reserved for extensive skull base defects not amenable to the endoscopic approach.

\section{References}

1. Dagi FT. Management of cerebrospinal fluid leaks. In: Schmidek HH, Roberts DW. Schmidek \& Sweet Operative Neurosurgical Techniques: Indications, Methods, and Results, vol. 1 5th ed., Philadelphia: Saunders Elsevier; 2006. pp 130-pp 145.

2. Thompson SC. The cerebro-spinal fluid; its spontaneous escape from the nose: with observations on its composition and function in the human subject. London: Cassell \& Company; 1899.

3. Miller CA. A case of hydrocephalus chronicus, with some unusual symptoms and appearance on dissection. Trans Med Chir Soc Edinb 1826;2:243-248.

4. Dandy W. Pneumocephalus (intracranial pneumatocele or aerocele). Archives of Surgery 1926;12(5):949-982. DOI: 10.1001/ archsurg.1926.01130050003001.

5. Cairns H. Injuries of the frontal and ethamoidal sinus with special reference to cerebrospinal fluid rhinorrhea and aerocele. J Laryngol Otol 1937;52(9):589-623. DOI: 10.1017/S0022215100043991.

6. Schroeder MC. Meningitis due to posttraumatic cerebro-spinal rhinorrhea. Arch Otolaryngol 1944;40(3):206-207. DOI: 10.1001/ archotol.1944.00680020260013.

7. Schlosser RJ, Bolger WE. Endoscopic management of cerebrospinal fluid rhinorrhea. Otolaryngol Clin N Am 2006;39(3):523-538. DOI: 10.1016/j.otc.2006.01.001.

8. Vishwakarma R, Patel K. Endoscopic surgical approach to CSF rhinorrhoea-analysis of 30 cases. International Journal of Science and Research 2014;3(1)20-24.

9. Lindstrom DR, Toohill RJ, LoehrlTA, etal. Management of cerebrospinal fluid rhinorrhea: the medical college of wisconsin experience. Laryngoscope 2004;114(6):969-974. DOI: 10.1097/00005537200406000-00003.

10. Mishra SK, Mathew GA. Endoscopic repair of CSF rhinorrhea: An institutional experience Iranian. Journal of Otorhinolaryngology 2016;28(1). DOI: 10.1007/s11033-016-3965-3Serial no. 84.

11. Mahammadi S, Daneshi A. Endoscopic management of cerebrospinal fluid rhinorrhea from anterior skull base defects. Medical Journal of the Islamic Republic of Iran 2008;22(1):12-16.

12. Cappabianca P, de Divitis O, Esposito F, et al. Endoscopic skull base instrumentation. In Anand VK, Schwartz TH, eds. Practical endoscopic skull base surgery. San Diego, Oxford, Brisbane: Plural Publishing Inc; 2007. pp. 45-56.

13. Senior $A B$, Derek L. Hydroscopy: application to pituitary surgery in: Transnasal Endoscopic Skull Base and Brain Surgery; Tips and pearls by Aldo Cassol Stamm; PA. New York: Thieme; 2012. pp. 3-14.

14. Clark $D$, Bullock $P$, Hui $T$, et al. Benign intracranial hypertension: a cause of CSF rhinorrhoea. J Neurol Neurosurg Psychiatry 1994;57(7): 847-849. DOI: 10.1136/jnnp.57.7.847.

15. Cappabianca P, Cavallo LM, Solari D, et al. Endoscopic endonasal surgery for pituitary adenomas. World Neurosurg 2014;82(6S):S3-S11. DOI: 10.1016/j.wneu.2014.07.019.

16. Lorenz RR, Dean RL, Hurley DB, et al. Endoscopic reconstruction of anterior and middle cranial fossa defects using acellular dermal allograft. Laryngoscope 2003;113(3):496-501. DOI: 10.1097/00005537200303000-00019. 